\title{
Observations on the adaptation of induced motion
}

\author{
ROBERT B. POST and LORI A. LOTT \\ University of California, Davis, California
}

\begin{abstract}
Induced motion (IM) was measured before and after a 10-min adaptation period during which subjects viewed the IM display without judging IM magnitude. The inducing stimulus was a rectangle, which contains both horizontal and vertical reference detail. The magnitude of IM was significantly lower following the adaptation period. This result is inconsistent with the hypothesis that adaptation of IM represents an instance of perceptual learning wherein the contribution of relative motion to motion perception is reduced. In a separate study, similar results were obtained when the inducing stimulus was a single vertical bar presented either to the left or to the right of the fixation stimulus. In addition, adaptation was obtained when the location of the inducing bar was changed during test measures, demonstrating that this effect is not specific to the retinal locus of the adaptation stimulus.
\end{abstract}

Induced motion (IM) is the apparent motion of a visual stimulus in the direction opposite the real motion of other visual stimuli. A wide variety of causes have been proposed to account for IM, ranging from the activity of neuronal units that respond to the motion of one visual stimulus relative to another, to the possibility that relative motion is a learned source of motion perception information (for a review, see Reinhardt-Rutland, 1988).

Given the diversity of proposed mechanisms for IM, it is potentially useful to investigate whether IM adapts, in that the characteristics of the adaptation might provide a basis for selecting among the various causal hypotheses. For example, if IM results from neuronal units responsive to relative image motion, any adaptation of IM (AIM) might be expected to dissipate rapidly in a manner similar to the motion aftereffect. Alternately, if IM results from the learning of relative motion information, the dissipation of AIM might be expected to take more time.

AIM was investigated by Wallach, Bacon, and Schulman (1978), who used a slant matching technique to study the effect of prolonged viewing of IM. ${ }^{1}$ In their study, subjects tracked a vertically oscillating spot against a background of long vertical bars oscillating horizontally. The apparent path of the vertically moving spot was measured before and after a 10-min adaptation period during which the vertical motion of the fixated spot was discontinued, while the background continued to move. Following the adaptation period, the spot appeared to move at less of a slant from vertical, indicating that the magnitude of IM was reduced.

In one condition in which horizontal bars were added to the background pattern, no AIM was measured. This

\footnotetext{
We are grateful to Marc Chaderjian for helpful comments on the research and manuscript. This work was supported in part by University of California Grant D-1832 to Robert B. Post. Correspondence may be addressed to Robert B. Post at the Department of Psychology, University of California, Davis, CA 95616.
}

was not interpreted as a failure to obtain an adaptation effect, however. Rather, it was suggested that this finding demonstrated that AIM resulted from a reduced contribution of relative motion information to motion perception. Specifically, despite the fact that subjects had been exposed to only one axis of relative motion during the adaptation period, it was proposed that the contribution of relative motion was decreased for all directions of stimulus motion. That is, the sensations of both horizontal and vertical components of stimulus motion were reduced similarly, thereby producing no change in the apparent slope of the motion path.

Wallach et al. (1978) discussed their findings within the general context of the role of learning in motion perception, suggesting that AIM was an instance wherein cue conflict leads to perceptual learning (i.e., learning that relative motion information no longer contributes as much to motion perception), rather than a sensory adaptation phenomenon. Consistent with this proposal was the finding that AIM persisted for at least 10 min following the adaptation period.

The analysis of results offered by Wallach et al. (1978) is interesting for two reasons. First, it supports the hypothesis that IM reflects the contribution of a learningbased mechanism to motion perception, in distinction to "low-level" mechanisms. Second, it predicts that no AIM should be obtained with an oscillating rectangular inducer stimulus similar to that commonly employed in IM studies (Reinhardt-Rutland, 1988), because rectangles contain horizontal elements. For these reasons, we examined whether or not AIM occurs with a rectangular inducing stimulus.

\section{EXPERIMENT 1}

\section{Method}

Fifteen subjects, 13 female and 2 male, participated. They were 19 to 34 years old, with a minimum $20 / 25$ visual acuity. The sub- 
jects judged the apparent slope of the motion path of a vertically oscillating fixation spot presented within a horizontally oscillating rectangular inducing stimulus. Both the fixation spot and the inducing stimulus were presented on a hemicylindrical projection screen (radius $=50 \mathrm{~cm}$ ). All observations were binocular, with the head stabilized by a chinrest. The rectangular inducing stimulus (outer edges) subtended $8.6^{\circ}$ vertically by $14^{\circ}$ horizontally. The edges of the rectangle subtended $.4^{\circ}$. The inducer oscillated sinusoidally through $7^{\circ}$ during $3 \mathrm{sec}$, yielding an inducing stimulus frequency of $.33 \mathrm{~Hz}$. This frequency was selected because it has been shown to produce robust IM (Post, Chi, Heckmann, \& Chaderjian, 1989). The fixation stimulus underwent vertical oscillations yoked to the horizontal oscillations of the inducing stimulus through the same spatial extent.

The subjects tracked the vertically oscillating fixation stimulus and indicated when they were ready to match the slope of the comparison stimulus to the perceived slant of the motion path of the tracked stimulus. At this time, a comparison stimulus consisting of a rotatable bar was illuminated and the tracked stimulus was occluded. The subjects grasped the bar and rotated it to the perceived slant of the previously viewed moving spot, and the slope was recorded by the experimenter. Five such judgments were performed both before and after the adaptation period. The adaptation period consisted of 10 min during which the subjects fixated the spot stimulus.

Two sessions were conducted on consecutive days (Day 1 and Day 2) to determine whether any AIM obtained during the first session was measurable $24 \mathrm{~h}$ later. Eight subjects participated first in a condition in which the fixation spot remained stationary (stationary adaptation; Day 1) and then in the second experimental condition, adapted to a moving fixation stimulus (moving adaptation; Day 2). For 7 subjects, the order of the adaptation conditions was reversed.

\section{Results and Discussion}

The magnitude of the horizontal component of IM was derived from each of the slant judgments, and the mean of the five measures in each condition for each subject was used in further evaluation. These values are presented in Table 1, along with the data expressed as degrees of apparent tilt of the motion path of the fixation stimulus from vertical. ${ }^{2}$ A $2 \times 2 \times 2$ (order of adaptation condition $X$ adaptation condition $X$ pre- or postadaptation measure) analysis of variance (ANOVA) indicated that IM decreased significantly between the pre- and postadaptation measures $[F(1,13)=7.79, p<.025]$. Mean IM magnitude in the preadaptation measures was $3.37^{\circ}\left( \pm 1.44^{\circ}\right)$; this decreased to $2.77^{\circ}\left( \pm 1.49^{\circ}\right)$ following adaptation. AIM was equal for moving and stationary adaptation conditions, as is evidenced by the lack of an interaction of the factors of pre- and postadaptation measures with adaptation stimulus $[F(1,13)=1.62, p>.1]$.

AIM obtained in the first session did not persist long enough to be measurable in the preadaptation measures of the second session, as is evidenced by the absence of a significant three-way interaction $[F(1,13)=3.35, p>.05]$. Mean preadaptation measures for Day 1 and Day 2 were $3.50^{\circ}\left( \pm 1.22^{\circ}\right)$ and $3.22\left( \pm 1.65^{\circ}\right)$, respectively. An analysis of the results of individual subjects confirmed that differences between Day 1 and Day 2 were not significant, for 9 of the 15 subjects demonstrated greater mean IM values in the Day 2 preadaptation measures.
Table 1

Pre- and Postadaptation Measures of Induced Motion (IM) Magnitude for Stationary and Moving Fixation Stimuli

\begin{tabular}{lcccccrr}
\hline \multirow{2}{*}{$\begin{array}{l}\text { Fixation } \\
\text { Stimulus }\end{array}$} & \multicolumn{2}{c}{ Preadaptation } & \multicolumn{2}{c}{ Postadaptation } & & \multicolumn{2}{c}{ Differences } \\
\cline { 2 - 4 } \cline { 7 - 8 } & $M$ & $S D$ & $M$ & $S D$ & & $M$ & $S D$ \\
\hline Stationary & 3.12 & 1.20 & 2.79 & 1.32 & .33 & .91 \\
Moving & 3.60 & 1.64 & 2.75 & 1.66 & .85 & 1.43 \\
& \multicolumn{7}{c}{ IM Magnitude (Degrees) } \\
Stationary & 23.40 & 8.00 & 21.00 & 8.90 & 2.40 & 6.56 \\
Moving & 26.00 & 9.80 & 20.40 & 10.96 & 5.60 & 9.61 \\
\hline
\end{tabular}

The amount of IM obtained in this experiment is less than that reported by Wallach et al. (1978). This difference owes most likely to the fact that Wallach et al.'s inducing stimulus was spatially more extensive $\left(90^{\circ} \times 64^{\circ}\right)$ than that used here $\left(8.6^{\circ} \times 14^{\circ}\right)$, as well as the fact that it consisted of a series of vertical stripes separated by only a few degrees. Therefore, Wallach et al.'s striped inducing stimulus brought inducing contours closer to the fixation stimulus than did our rectangular inducing stimulus. Both inducer size and adjacency have been demonstrated to have a large influence on the magnitude of IM (for a review, see Reinhardt-Rutland, 1988). Although there was less IM in the present study, it is interesting that the percent reduction obtained $(17.8 \%)$ is comparable to that reported (about $15 \%$ ) by Wallach et al.

The results of Experiment 1 contradict the hypothesis, proposed by Wallach et al. (1978), that AIM represents a decrease in the contribution of relative motion information to perceived motion. Rather, AIM is specific to the direction of inducer motion during adaptation. It is therefore similar to sensory adaptation phenomena such as the adaptation of directionally selective motion-analyzing units or channels in the nervous system (see, e.g., Pantle \& Sekuler, 1969), rather than a general learning phenomenon. In addition, the finding that AIM was eliminated during the delay between the first and second experimental sessions is inconsistent with the hypothesis that AIM represents the acquisition of new strategies by subjects for performing the slant matching task.

Because the preceding results suggested that AIM is direction-specific, we next examined whether AIM is specific to the retinal locus of the inducing stimulus, as is the case for examples of the adaptation of directionspecific motion-sensing mechanisms (see, e.g., Anstis \& Gregory, 1965).

\section{EXPERIMENT 2}

\section{Method}

Thirty-two subjects, 19 female and 13 male, participated. They were 17 to 23 years old, with a minimum 20/25 visual acuity. The subjects judged the apparent slope of a vertically moving fixation stimulus presented against a background consisting of a portion of the previously described inducing stimulus. Specifically, the horizontal edges were removed, as well as either the left or the right verti- 
Table 2

Pre- and Postadaptation Measures of Induced Motion (IM) Magnitude for Left and Right Inducing Stimuli

\begin{tabular}{|c|c|c|c|c|c|c|c|c|c|c|c|c|}
\hline & \multicolumn{4}{|c|}{ Preadaptation } & \multicolumn{4}{|c|}{ Postadaptation } & \multicolumn{4}{|c|}{ Differences } \\
\hline & \multicolumn{2}{|c|}{ Left Bar } & \multicolumn{2}{|c|}{ Right Bar } & \multicolumn{2}{|c|}{ Left Bar } & \multicolumn{2}{|c|}{ Right Bar } & \multicolumn{2}{|c|}{ Left Bar } & \multicolumn{2}{|c|}{ Right Bar } \\
\hline & $M$ & $S D$ & $M$ & $S D$ & $M$ & $S D$ & $M$ & $S D$ & $M$ & $S D$ & $M$ & $S D$ \\
\hline $\begin{array}{l}\text { IM magni } \\
\text { IM tiltt }\end{array}$ & $\begin{array}{r}1.89 \\
14.17\end{array}$ & $\begin{array}{l}1.09 \\
7.54\end{array}$ & $\begin{array}{r}2.06 \\
14.96\end{array}$ & $\begin{array}{r}1.73 \\
10.18\end{array}$ & $\begin{array}{r}1.38 \\
10.55\end{array}$ & $\begin{array}{l}1.05 \\
7.64\end{array}$ & $\begin{array}{r}1.59 \\
11.48\end{array}$ & $\begin{array}{l}1.59 \\
9.80\end{array}$ & $\begin{array}{c}.51 \\
3.62\end{array}$ & $\begin{array}{l}1.09 \\
7.39\end{array}$ & $\begin{array}{c}.47 \\
3.48\end{array}$ & $\begin{array}{l}1.53 \\
9.86\end{array}$ \\
\hline
\end{tabular}

*Degrees. †Degrees from vertical.

cal edge, so that the inducing stimulus consisted of either the left or the right vertical bar of the original inducing rectangular stimulus.

Four IM measures were obtained for each inducing stimulus both before and after an adaptation period. During adaptation, the subjects fixated the vertically oscillating spot for $10 \mathrm{~min}$ in the presence of a horizontally moving vertical edge located to the right of the fixation spot. Following this period, IM was measured with an inducing stimulus either identical to that present during the adaptation period or mirror symmetrical with regard to fixation. Half of the subjects performed these judgments initially for the left inducing stimulus, and the other half viewed the right inducing stimulus first.

\section{Results}

The magnitude of the horizontal component of IM was derived from each of the slant judgments, and the mean of the four measures in each condition for each subject was used in further evaluation. These values are presented in Table 2, along with the data expressed as degrees of apparent tilt of the motion path of the fixation stimulus from vertical. A $2 \times 2 \times 2$ (location of inducing stimulus tested first $\times$ location of inducing stimulus $\times$ pre- vs. postadaptation measure) ANOVA indicated that IM decreased significantly between the pre- and postadaptation measures $[F(1,30)=9.02, p<.01]$. Mean IM magnitude prior to the adaptation period was $1.97^{\circ}( \pm 1.44)$ and $1.49^{\circ}\left( \pm 1.36^{\circ}\right)$ afterward. There was, however, no significant interaction between the pre- and postadaptation measures and the location of the inducing stimulus $[F(1,30)=.009, p>.1]$. The lack of an interaction indicates that AIM was not specific to the retinal locus of the inducing stimulus present during adaptation. The smaller amount of IM in this study relative to that in Experiment 1 was an expected result of the reduction in the size of the inducing stimulus.

\section{GENERAL DISCUSSION}

The primary finding in this paper is that AIM occurs with a rectangular inducing stimulus typical of that used in many IM studies. This is inconsistent with the suggestion of Wallach et al. (1978) that AIM represents a reduction of the contribution of relative motion information to motion perception. Such an explanation predicts that AIM should not be apparent if the test stimulus contains both horizontal and vertical reference detail, because the diminished motion perception produced by the adaptation period should apply to both the horizontal and the verti- cal components of perceived motion. Therefore, no net change in the apparent slope of the motion path of a fixation stimulus would be expected. However, the present results indicate that AIM represents an adaptation of motion perception along the same axis as that of the motion of the inducing stimulus, and no apparent adaptation of motion perception along other dimensions.

The results also suggest that AIM does not reflect a shift in the strategy used by subjects in judging perceived motion, for it dissipates between Day 1 and Day 2. Wallach et al. (1978) had reported that AIM persists for at least $10 \mathrm{~min}$. If the basis of the adaptation effect was the acquisition of a new strategy for performing slant-matching judgments, it might be expected that the adaptation effect would persist for a longer period.

In addition, AIM was found to be independent of the retinal location of the adaptation stimulus. This result suggests that AIM does not reflect the adaptation of directionsensitive mechanisms such as those mediating the motion aftereffect, for such effects are specific to the retinal locus of stimulation (see, e.g., Anstis and Gregory, 1965).

Howard (1982) has distinguished between different frames of reference that can be used in interpreting motion of visual stimuli ranging from lower to higher levels of visual analysis (i.e., retinocentric, oculocentric, egocentric, and exocentric). The results of Experiment 1 suggest that a higher order exocentric explanation (i.e., the diminished effectiveness of configuration change as a result of perceptual learning) cannot fully account for AIM. On the other hand, the present Experiment 2 suggests that the lowest level, or retinocentric frame by itself (i.e., adaptation of direction-specific retinal neurons) cannot account for AIM. We suggest that a medium level of analysis (oculocentric or egocentric) might be responsible for AIM.

Why do our results contradict those of Wallach et al. (1978), who obtained no AIM when horizontal reference detail was added to their inducing stimulus? It is possible that the difference between studies owes to the differences in the inducing stimuli previously noted. In addition, it should be noted that Wallach et al. also altered the ratio of fixation stimulus motion to inducing stimulus motion from $1: 1$ to $.58: 1$ in the critical condition in which horizontal lines were added. Perhaps this additional methodological change was responsible for the lack of AIM in that condition. Regardless of what is responsible for the differ- 
ent results obtained in the two studies, the present experiments demonstrate significant AIM under conditions in which horizontal reference detail is available.

\section{REFERENCES}

ANSTIS, S. M., \& GREGoRY, R. L. (1965). The aftereffect of seen movement: The role of retinal stimulation and of eye movements. Quarterly Journal of Experimental Psychology, 17, 173-174.

GOGEL, W. C., \& TIETZ, J. D. (1976). Adjacency and attention as determinants of induced motion. Vision Research, 16, 839-845.

HowARD, I. P. (1982). Human visual orientation. New York: Wiley.

Pantle, A., \&ekuler, R. (1969). Contrast response of human visual mechanisms sensitive to orientation and direction of motion. Vision Research, 9, 397-406.

Post, R. B., Chi, D., Heckmann, T., \& Chaderian, M. (1989). A reevaluation of the effect of velocity on induced motion. Perception \& Psychophysics, 45, 411-416.

REINHARDT-RutLAND, A. H. (1988). Induced movement in the visual modality: An overview. Psychological Bulletin, 103, 57-71.

Wallach, H., Bacon, J., \& SChulman, P. (1978). Adaptation in mo- tion perception: Alteration of induced motion. Perception \& Psychophysics, 24, 509-514.

\section{NOTES}

1. The magnitude of IM may be measured through having subjects fixate a stimulus that oscillates vertically, while another stimulus undergoes correlated oscillations horizontally. Under these conditions, the slope of the path of the tracked stimulus appears to be shifted from vertical as the result of perceptual summation of an illusory horizontal motion vector (IM) with the real vertical motion vector (Gogel \& Tietz, 1976). The apparent slopes may be measured through having subjects adjust the slant of a comparison stimulus, and the magnitude of the IM component may be derived from their settings. This technique is referred to here as the slant matching technique.

2. The tabled values of IM refer to the horizontal vector of illusory motion. Values presented as tilt in degrees from vertical do not directly express the magnitude of IM, because the horizontal illusory motion vector varies as a function of the tangent of the tilt angle.

(Manuscript received August 31, 1990; revision accepted for publication November 27, 1990.) 